\title{
A study on the influence of regional medical service design on the life of aged people
}

\author{
Zhong Lei, Yang Chang, JIANG Hong-ling, Yang Yue \\ Tianjin University of Technology,Tianjin,300384,China
}

Keywords: public self-service design; emotional design; aging population

\begin{abstract}
In recent years, the number of Chinese aging population continues to increase, the design of public self-service aging population can effectively improve the social work efficiency based on social cost savings, solve some of the problems of an aging society. Through the analysis and summary of the needs of the elderly population, at the same time, the current situation of the use of public self-service facilities in the region are studied, and the corresponding design methods and principles. Finally, through the investigation and study for the aging of regional public service design background and providing experience, design of regional public self-service to play better, to provide high-quality personalized service for the elderly.
\end{abstract}

\section{Introduction}

The acceleration of population aging, the elderly to the medical service demand, but the market of medical needle self-service design for the elderly are very scarce, most of the medical service products does not reflect the characteristics of emotional design and targeted, difficult operation, unclear instructions easy to cause operational errors between pay no attention to the emotional communication with the users. In view of the psychological and physiological characteristics of the elderly, the design of the barrier free medical service is the focus of the designer.

\section{The summary of relevant concepts}

\subsection{Public service design concept}

The design of public self-service is to provide the creative development of the public life and the public service field, and provide different levels of innovative methods and design knowledge for the user's own technology platform.

\subsection{Aging related concept}

WHO for the elderly is defined as 65 to 74 years of age are young adults, aged from 75 to 90 years old is the real elderly, aged from 90 to 120 years old. Over 60 years old in china.

\section{The aging population analysis}

\subsection{The present situation of aging}

The phenomenon of aging population is becoming more and more prominent in our country, so most of the resources of the hospital will be used for the elderly. Adjust the allocation of medical resources is imperative.Urgent needs of older products and huge product design services are not in place. Health care products of a single design is not good, can not meet the needs of the elderly, which requires medical institutions to provide more medical services for the elderly population, and for the elderly to make targeted design, so as to help improve the quality of life of the aging population.

\subsection{Physiological analysis of aging population}

(1) Decreased self-care ability.

(2) Resistance, decreased body immunity, decline in the physiological system, hearing, visual decline.

(3) Degeneration of central nervous system and decrease 
of response ability.

(4) Adaptive decline.

\subsection{Analysis of psychological needs of aging population}

The elderly lack of motivation to learn new things and requirements, perception of aging will increase the probability of the elderly to make new mistakes, often negative emotions, such as anxiety, remorse, extreme tension. Old people have a fear of high tech products. At the same time, Older people have higher psychological security needs.

\section{Current situation and case study of regional medical public service design}

\subsection{Domestic and international status quo of regional} medical public service design

The situation of our country is different from that of the developed countries, the aging of the developed countries is basically synchronized with the modernization. Although China's rapid economic development, but when entering the aging, the overall level of GDP per capita is still low.In recent years, with the support of national policy and social public service, the design of domestic regional healthcare is under construction and improvement, but there are still many problems existing in the design.

\subsection{The domestic case analysis}

In this paper, through the investigation of the present situation of the self-service medical equipment in Tianjin First Central Hospital, the existing problems are analyzed and summarized.

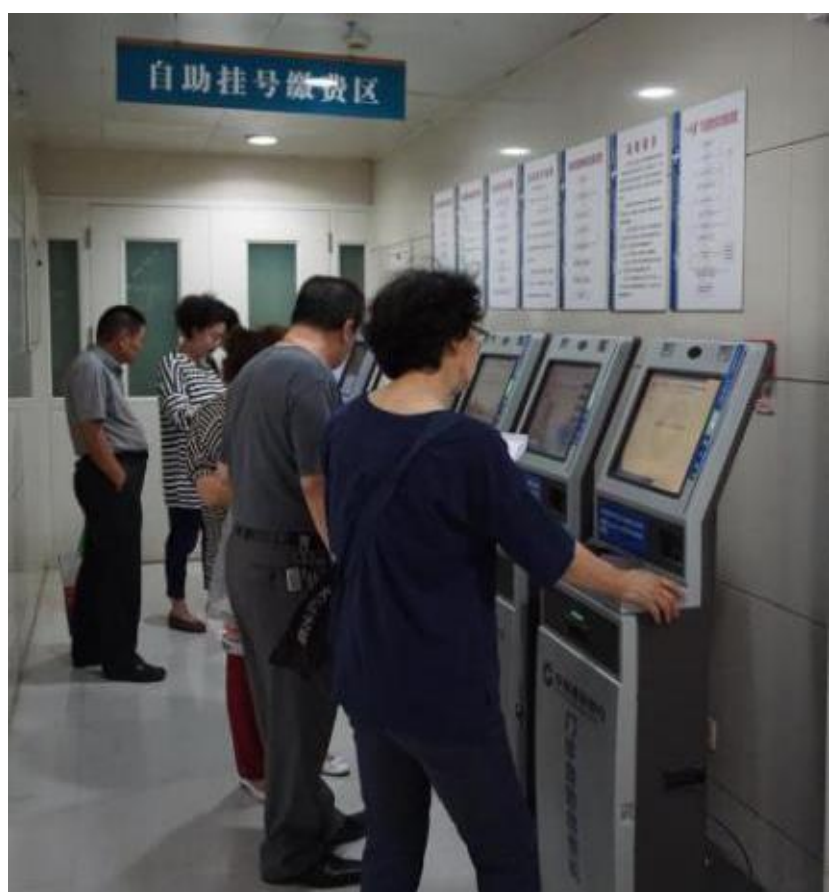

Fig.1 First central hospital self-service machine



Fig.2 Self service take a single card slot

Regional public self-service facilities of the independent use of space within the hospital less, arrange the position placed scattered and not convenient to use (Figure 1), and there is no independent safe use area,users are reluctant to try self-service facilities.

The elderly is a special group, in the design should fully reflect the emotional care, medical self-service facilities currently has simple shape, cold machine, suggesting the lack of humanized use process (Figure 2), a waste of time for medical treatment, the elderly users a respectful distance. The interface is mostly in the form of text, it will bring inconvenience to the elderly who do not have the background of cultural knowledge. At the same time, the text size is too small, the interface is too cumbersome to 
convey information to increase the use of the elderly and the fear of science and technology.

\subsection{Case study abroad}

Japanese hospital is an appointment system, do not need a temporary queue, thus diverting the crowd. So that the hospital queue, registration difficulties have been effectively controlled .

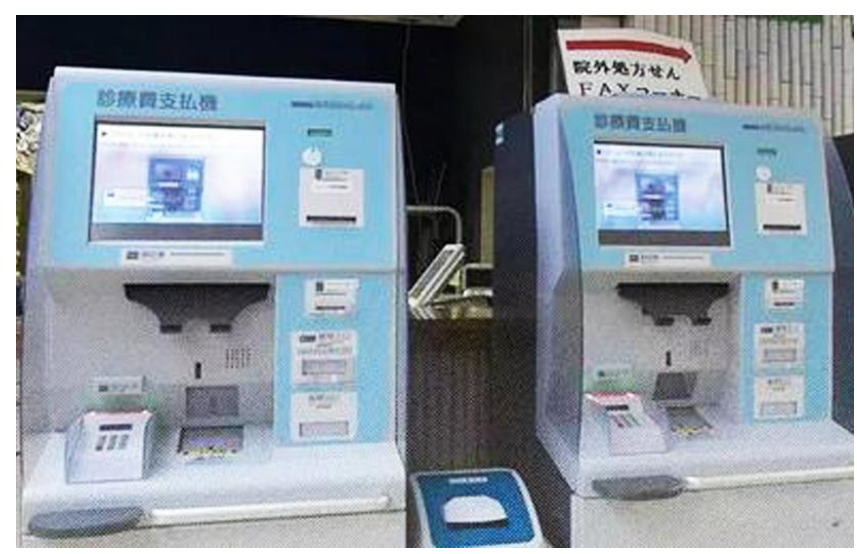

Fig.3 Japanese hospital self-service payment machine

Japan Hospital self-service payment without time limit (Figure 3), health insurance card storing consumer records, as long as you can complete the payment within the specified time, avoid queuing congestion and the elderly stand for a long time, waiting for the fatigue; The hospital did not set the drug point, so the purchase of drugs to the appropriate pharmacy, effectively avoiding the possibility of making a difference in the middle of the hospital.At the same time, also reduced the flow of people in the hospital, the doctor provides more space for elderly patients.

\section{The design principles of regional public self service design based on aging population}

Universal design is a compromise between differential design and uniform design. Aiming at the characteristics of the physiological and psychological needs of the elderly population, the design method of the universal interface for aging population is designed.

First, the operation interface design of the elderly population should be in line with its own characteristics, at the same time, can not affect the use of other suitable people; design should be simple and easy to understand, so that the elderly can operate alone. In the interface design, for the elderly to provide clear information for the operation, but also timely feedback letter to remind the operation completed.

Second, the interface design for the elderly, should be fully taken into account the different types of elderly people, such as the lower level of education for older people, while placing the text and image information on the operating interface. For the elderly people with weak eyesight can increase voice prompts, especially in the operation of the error will be issued a warning to remind the elderly to cancel the wrong operation.

Third, the arrangement design interface button should conform to the habits of the elderly, reduce repetitive movements, avoid the troublesome operation, main keys and display interface should be clear and easy to understand, try to complete a one-time operation; key size and spacing should be considered reasonable arrangements for the elderly of the actual situation, to facilitate the elderly.

\section{The application of emotional design principles in the design of self service for the elderly}

There is no product to meet all the needs of the people, because everyone's views on a product are not the same, in addition to rational analysis, while there are emotional factors. The three kind of behavior is the interaction, the mutual adjustment, affects our behavior in different ways. The level of instinct is the most primitive part of the brain. In this level, the human response has both generality and difference.Therefore, in the design of public service in medical service, we should design according to the needs and preferences of the elderly. The level of instinct is related to the initial response of human beings, so it can be improved from the visual, auditory, tactile and other physiological characteristics. The appearance of the product can be sleek curve design, erase the design of the cold line, each step will have a certain voice prompts.

The behavior level is usually related to the use of the product and the operating experience, including product features, performance and availability. To observe the potential problems existing in the operation of the elderly and to improve them. Change the frustration of older people when using the product. People oriented design, with 
simple and clever flash images or sounds, so that the elderly management[J].2014. need to effectively integrate information. The design of a truly effective and good service for the elderly.

The level of reflection is related to the meaning of information, culture and products. It usually determines the individual's overall perception of a product. At this level, the user will recall the previous use of the product process, product defects can be enlarged or ignored, it will break the original proportion. So the designer in the design of a product, through the product semantics to help users resolve grievances, to provide a positive experience for the user.

\section{Outlook and summary}

In the near future, China's aging has become a trend. The physiological characteristics and grasp the psychological demands of aging population, designed to meet the needs of medical self-service products to meet the aging population diverse health care needs, can fully cover all the health care needs of the elderly, can make the experience better public services to social health, improve their late years of life.

\section{References}

[1] Zhu Guo-hong, Lu Yuan. The development potential of China's aging industry: a demand side analysis of [J]. Population studies, 2001 (6).

[2] Li Zi-cheng,Wang Wen-ni. The present situation, problems and Countermeasures of the development of the aging service industry[J].Macro economic management,2013(5): 70-72.

[3] LI Chao, LI Yin. Thinking about the design of urban public facilities $[\mathrm{J}]$. Journal of Zhengzhou Institute of Aeronautics Industry Management (SOCIAL SCIENCE EDITION), 2007, 26 (5): 194-195.

[4] LI Yong-chun, LIU Bei-lei. Comparison of universal design and personalized design[J].Creativity and design. 2010.01

[5] LI Ze. The general design of the control interface of the household medical devices in the elderly[J].Engineering Science and technology II period.2011.51.

[6] Ding Zheng-yu, Zhu Zhao-feng, Shen Wei, Yang Xiao-jun, Chen Zhao-jiong. Application and Prospect of medical self service terminal equipment in hospital 